\title{
212.
}

\section{A MEMOIR ON THE PROBLEM OF DISTURBED ELLIPTIC MOTION.}

[From the Memoirs of the Royal Astronomical Society, vol. xxviI., 1859, pp. 1-29. Read March 9, 1858.]

I VENTURE to take up the problem of disturbed elliptic motion, for the sake of a further elaboration of the analytical theory. The points which present difficulty are the measurement of longitudes in the varying plane of the orbit, and (in the lunar theory) the determination of the position of the orbit by reference to the varying plane of the sun's orbit; it is, in memoirs and works on the lunar and planetary theories, often difficult to discover where or how (or whether at all) account is taken of these variations, and the analytical mode of treatment is for the most part very imperfect. I must except always Hansen's Fundamenta Nova [investigationis orbitce verce quam Luna perlustrat, Gotha 1838] where the points referred to are treated in a perfectly rigorous manner. There is, however, a want of clearness in the form under which his investigations are presented; and the comprehension of them is greatly facilitated by Jacobi's remarks, published under the title "Auszug zweier Schreiben des Prof. Jacobi an Herrn Director Hansen" (Crelle, t. xuII. pp. 12-31 (1851)). Jacobi observes that the integration of Hansen's system of differential equations introduces seven arbitrary constants, which, in the expressions for the coordinates referred to fixed axes, reduce themselves to six. The seventh constant, neglecting the disturbing forces, is in fact a constant which determines the position in the orbit of the arbitrary origin from which the longitudes in orbit are reckoned. I have, in my paper "On Hansen's Lunar Theory," Quarterly Mathematical Journal, vol. I. pp. 112-125 (1855), [163], termed this origin "the departure-point," and longitudes measured from it "departures." The seventh constant may be taken to be the departure of the node. I reproduce in the present memoir the explanation of what is meant by the departure when the plane of the orbit is variable. If the problem is treated by the method of the variation of the elements, the seventh constant becomes, like the other elements, variable; and we have 
thus a seventh variable element, the departure of the node. The element just referred to (the departure of the node) forms, with the longitude of the node and the inclination, a group of three elements, which determine the position of the orbit and of the departure-point. The coordinates of the planet are in the first instance taken to be the radius vector, longitude, and latitude; but the before-mentioned three elements being considered as given, the position of the planet depends only on the radius vector and the departure. These may be then expressed in terms of the remaining four elements; as to the choice of these four elements, it is to be remarked that there is one element which only enters through the mean anomaly, and that there is great convenience in representing with Hansen the mean anomaly by a single letter; and that in the various formulæ we may use, in the place of the element implicitly involved in the mean anomaly, the mean anomaly itself, or treat the mean anomaly as an element; the four elements may be taken to be the semi-axis major, the eccentricity, the mean anomaly, and the departure of the pericentre. And joining to these the before-mentioned three elements, we have the system of elements represented in the memoir by $a, e, g, \varpi, \sigma, \theta, \phi$. It has been assumed so far that the three elements determine the position of the orbit and departure-point in reference to a fixed plane and origin of longitudes; but we may suppose more generally that, instead of the fixed plane and origin of longitudes, we have a variable plane or orbit of reference and a departurepoint in this variable orbit of reference. The quantities which determine the orbit of reference and departure-point are naturally taken to be the departure of the node, longitude of the node, and inclination; these are assumed to be given functions of the time, and they are in the memoir represented by $\sigma^{\prime}, \theta^{\prime}, \phi^{\prime}$. The three elements of the planet's orbit (viz. departure of node, longitude of node, and inclination) in relation to the orbit of reference and departure-point therein, are in the memoir represented by $\Sigma, \Theta, \Phi$, and the system of elements ultimately adopted is therefore $a, e, g, \sigma, \Sigma, \Theta, \Phi$. I obtain formulæ for the variations of these elements under two different modes of expression of the disturbing function: first, when the disturbing function is expressed in terms of the radius vector and departure and of the three elements $\Sigma, \Theta, \Phi$; secondly, when the disturbing function is expressed in terms of the seven elements $a, e, g, \varpi, \Sigma, \Theta, \Phi$. The establishment of the two sets of formulæ just referred to constitutes the chief object of the memoir; but the memoir contains some other investigations and formulæ in relation to the general subject.

The coordinates of the planet are

$$
\begin{aligned}
& r \text {, the radius vector, } \\
& v \text {, the longitude, } \\
& y \text {, the latitude. }
\end{aligned}
$$

The attractive force at distance unity is for convenience represented by $n^{2} a^{3}$, which denotes, therefore, an absolute constant; but the significations of $n$ and $a$ are not yet defined.

The disturbing function, as used by Lagrange, is denoted by $\Omega$, that is $\Omega=-R$, if $R$ be the disturbing function of the Mécanique Céleste. 
The equations of motion are

$$
\begin{array}{lr}
\frac{d}{d t} \frac{d r}{d t}-r \cos ^{2} y\left(\frac{d v}{d t}\right)^{2}-r\left(\frac{d y}{d t}\right)^{2}+\frac{n^{2} a^{3}}{r^{2}} & =\frac{d \Omega}{d r}, \\
\frac{d}{d t}\left(r^{2} \cos ^{2} y \frac{d v}{d t}\right) & =\frac{d \Omega}{d v}, \\
\frac{d}{d t}\left(r^{2} \frac{d y}{d t}\right)+r^{2} \cos y \sin y\left(\frac{d v}{d t}\right)^{2} & =\frac{d \Omega}{d y},
\end{array}
$$

where $\Omega$ is regarded as a function of $r, v, y$, or (as this may be expressed) where $\Omega=\Omega(r, v, y)$.

If we neglect the disturbing forces, the planet moves in an ellipse; and taking $a$ to represent the semi-axis major, the mean motion will be $n$. The mean anomaly, which I call $g$, will be a function of the form $n t+c$; but as $c$ only enters through $g$, it will be convenient to use the mean anomaly $g$ (considered as implicitly involving an arbitrary constant $c$ ) in the place of an element, and I write

$$
\begin{aligned}
& a \text {, the semi-axis major, } \\
& e \text {, the eccentricity, } \\
& g \text {, the mean anomaly, } \\
& \theta \text {, the longitude of node, } \\
& \phi \text {, the inclination, } \\
& \boldsymbol{\tau} \text {, the distance of pericentre from node. }
\end{aligned}
$$

I assume also

$$
\begin{aligned}
& f \text {, the true anomaly, } \\
& z \text {, the distance of planet from node, } \\
& x \text {, the reduced distance from node. }
\end{aligned}
$$

We have then $r$ and $f$ given functions of $t$ and the elements, viz. we may write

$$
\begin{aligned}
& r=a \text { elqr }(e, g), \\
& f=\text { elta }(e, g),
\end{aligned}
$$

(read elqr. elliptic quotient radius, and elta. elliptic anomaly). These values satisfy $r=\frac{a\left(1-e^{2}\right)}{1+e \cos f}$. Moreover $z, x, y$, are the hypothenuse, base, and perpendicular of a right-angled spherical triangle, the base angle whereof is $\phi$; the equations which connect these quantities are therefore

$$
\begin{aligned}
& \tan x=\tan z \cos \phi, \\
& \sin y=\sin z \sin \phi, \\
& \tan y=\sin x \tan \phi, \\
& \cos z=\cos x \cos y,
\end{aligned}
$$


equivalent, of course, to two equations. The first and second of them give in fact $x, y$, in terms of $z$ and $\phi$.

The value of $z$ is

$$
z=\tau+f
$$

so that $x$ and $y$ are given functions, and the longitude $v$ is given in terms of $x$ and $\theta$ by the equation

$$
v=x+\theta,
$$

and consequently the three coordinates $r, v, y$, are by the system of equations given in terms of $t$ and the elements.

From the equations which connect $z, x, y, \phi$, treating all these quantities as variable we deduce

$$
\begin{aligned}
& \sec ^{2} x d x=\cos \phi \sec ^{2} z d z-\tan z \sin \phi d \phi, \\
& \cos y d y=\sin \phi \cos z d z+\sin z \cos \phi d \phi, \\
& \sec ^{2} y d y=\tan \phi \cos x d x+\sin x \sec \phi d \phi, \\
& \sin z d z=\cos y \sin x d x+\cos x \sin y d y,
\end{aligned}
$$

equivalent of course to two equations; and the system is easily reduced to the more convenient form

$$
\begin{aligned}
& d x=\quad \cos \phi \sec ^{2} y d z-\tan z \cos ^{2} x \sin \phi d \phi, \\
& d y=\quad \sin \phi \cos x d z+\cos x \tan z \cos \phi d \phi, \\
& d x=\cot \phi \sec x \sec ^{2} y d y-\quad \tan z \operatorname{cosec} \phi d \phi \text {, } \\
& d z=\quad \cos \phi d x+\quad \cos x \sin \phi d \phi,
\end{aligned}
$$

joining to these equations the

$$
\begin{aligned}
& d z=d \tau+d f \\
& d v=d x+d \theta
\end{aligned}
$$

and considering at present the mere analytical forms, first if $d \phi=0, d \tau=0$, we have

$$
\begin{aligned}
& d x=\cos \phi \sec ^{2} y d z, \\
& d y=\sin \phi \cos x d z, \\
& d z=d f, \\
& d v=d x .
\end{aligned}
$$

Next, if $d y=0, d v=0$, we have

$$
\begin{aligned}
& d x=-\tan z \operatorname{cosec} \phi d \phi, \\
& d z=-\tan z \cot \phi d \phi, \\
& d z=\cos \phi d x, \\
& d x=-d \theta \\
& d z+\cos \phi d \theta=0 .
\end{aligned}
$$

I remark also that the equation, $\tan y=\sin x \tan \phi$, may be written in the form

$$
\cos ^{2} \phi \sec ^{2} y+\sin ^{2} \phi \cos ^{2} x=1 \text {. }
$$

C. III. 
The equations

$$
\begin{aligned}
& r=a \text { elqr }(e, g), \\
& f=\text { elta }(e, g),
\end{aligned}
$$

treating all the quantities as variable, give

$$
\begin{aligned}
& d r=\frac{a e \sin f}{\sqrt{1-e^{2}}} d g+\frac{1-e^{2}}{1+e \cos f} d a-a \cos f d e, \\
& d f=\frac{(1+e \cos f)^{2}}{\left(1-e^{2}\right)^{\frac{3}{2}}} d g+\frac{\sin f(2+e \cos f)}{1-e^{2}} d e,
\end{aligned}
$$

to which is to be joined

$$
d r=\frac{a e\left(1-e^{2}\right) \sin f}{(1+e \cos f)^{2}} d f+\frac{1-e^{2}}{1+e \cos f} d a+\frac{a\left(2 e-\overline{1+e^{2}} \cos f\right)}{(1+e \cos f)^{2}} d e,
$$

all which formulæ will be useful.

If we treat the elements as constant, then in the foregoing expressions for $d r$ and $d f$, we must attend only to the part involving $d g$, and must put this equal to $n d t$; the values first obtained for $d x, d y, d z, d v$, correspond to this assumption, and we have

$$
\begin{aligned}
& \frac{d r}{d t}=\frac{n a e \sin f}{\sqrt{1-e^{2}}} \\
& \frac{d f}{d t}=\frac{n a^{2} \sqrt{1-e^{2}}}{r^{2}} \\
& \frac{d z}{d t}=\frac{n a^{2} \sqrt{1-e^{2}}}{r^{2}} \\
& \frac{d x}{d t}=\cos \phi \sec ^{2} y \frac{n a^{2} \sqrt{1-e^{2}}}{r^{2}} \\
& \frac{d v}{d t}=\cos \phi \sec ^{2} y \frac{n a^{2} \sqrt{1-e^{2}}}{r^{2}} \\
& \frac{d y}{d t}=\sin \phi \cos x \frac{n a^{2} \sqrt{1-e^{2}}}{r^{2}}
\end{aligned}
$$

and we then deduce

$$
\begin{array}{ll}
\frac{d}{d t} \frac{d r}{d t} & =\frac{n a^{3} e \cos f}{r^{2}} \\
\frac{d}{d t}\left(r^{2} \cos ^{2} y \frac{d v}{d t}\right) & =0 \\
\frac{d}{d t}\left(r^{2} \frac{d y}{d t}\right) & =-\cos ^{2} \phi \sin y \sec ^{3} y \frac{n^{2} a^{4}\left(1-e^{2}\right)}{r^{4}}, \\
\frac{d}{d t}\left(\frac{d f}{d t}\right) & =-\frac{2 n^{2} a^{3} e \cos f}{r^{2}}
\end{array}
$$

values which satisfy the undisturbed equations. 
The disturbed equations may be dealt with in the usual manner by the method of the variation of the elements, and attending only to the variations of the elements we have

or, what is the same thing,

$$
\begin{aligned}
d r & =0, \\
d v & =0, \\
d y & =0, \\
d \frac{d r}{d t} & =\frac{d \Omega}{d r} d t, \\
d\left(r^{2} \cos ^{2} y \frac{d v}{d t}\right) & =\frac{d \Omega}{d v} d t, \\
d\left(r^{2} \frac{d y}{d t}\right) & =\frac{d \Omega}{d y} d t
\end{aligned}
$$

$$
\begin{aligned}
d r & =0, \\
d v & =0, \\
d y & =0 \\
d \frac{n a e \sin f}{\sqrt{1-e^{2}}} & =\frac{d \Omega}{d r} d t \\
d n a^{2} \sqrt{1-e^{2}} \cos \phi & =\frac{d \Omega}{d v} d t \\
d n a^{2} \sqrt{1-e^{2}} \sin \phi \cos x & =\frac{d \Omega}{d y} d t
\end{aligned}
$$

where as before $\Omega=\Omega(r, v, y)$.

In virtue of the relations $d v=0, d y=0$, we have the above-mentioned equations,

$$
\begin{aligned}
& d x=-\tan z \operatorname{cosec} \phi d \phi \\
& d z=-\tan z \cot \phi d \phi \\
& d z=\cos \phi d x \\
& d x=-d \theta \\
& d r \quad+\cos \phi d \theta=0
\end{aligned}
$$

we have

$$
\begin{aligned}
d \sin \phi \cos x & =-\sin \phi \sin x d x+\cos x \cos \phi d \phi, \\
& =\cos x \cos \phi \sec ^{2} z d \phi, \\
& =\sec x \cos \phi \sec ^{2} y d \phi ;
\end{aligned}
$$

and the last two equations for the variations become

$$
\begin{aligned}
& d n a^{2} \sqrt{1-e^{2}} \cos \phi \quad-n a^{2} \sqrt{1-e^{2}} \sin \phi d \phi \quad=\frac{d \Omega}{d v} d t, \\
& d n a^{2} \sqrt{1-e^{2}} \sin \phi \cos x+n a^{2} \sqrt{1-e^{2}} \sec x \cos \phi \sec ^{2} y d \phi=\frac{d \Omega}{d y} d t
\end{aligned}
$$


and attending to the equations $\cos ^{2} \phi \sec ^{2} y+\sin ^{2} \phi \cos ^{2} x=1$ we deduce at once

$$
\begin{array}{llr}
d n a^{2} \sqrt{1-e^{2}} & = & \cos \phi \sec ^{2} y \frac{d \Omega}{d v} d t+\sin \phi \cos x \frac{d \Omega}{d y} d t, \\
d \phi & =\frac{1}{n a^{2} \sqrt{1-e^{2}}}\left(-\sin \phi \cos ^{2} x \frac{d \Omega}{d v} d t+\cos \phi \cos x \frac{d \Omega}{d y} d t\right) .
\end{array}
$$

Now the position of the planet may be determined by the quantities $r, z, \theta, \phi$, or we may consider $\Omega$ as a function of the last-mentioned quantities. And if on the right-hand side $\Omega=\Omega(r, v, y)$ as before, the formulæ of transformation are

$$
\frac{d \Omega}{d r} d r+\frac{d \Omega}{d v} d v+\frac{d \Omega}{d y} d y=\frac{d \Omega}{d r} d r+\frac{d \Omega}{d z} d z+\frac{d \Omega}{d \theta} d \theta+\frac{d \Omega}{d \phi} d \phi
$$

where

$$
\begin{aligned}
& d v=\cos \phi \sec ^{2} y d z-\tan z \cos ^{2} x \sin \phi d \phi+d \theta \\
& d y=\sin \phi \cos x d z+\tan z \cos x \cos \phi d \phi
\end{aligned}
$$

and we have

$$
\begin{aligned}
& \frac{d \Omega}{d r}=\frac{d \Omega}{d r}, \\
& \frac{d \Omega}{d \theta}=\frac{d \Omega}{d v}, \\
& \frac{d \Omega}{d \phi}=\tan z\left(-\sin \phi \cos ^{2} x \frac{d \Omega}{d v}+\cos \phi \cos x \frac{d \Omega}{d y}\right), \\
& \frac{d \Omega}{d z}=\left(\cos \phi \sec ^{2} y \frac{d \Omega}{d v}+\sin \phi \cos x \frac{d \Omega}{d y}\right),
\end{aligned}
$$

where on the leit-hand side $\Omega=\Omega(r, z, \theta, \phi)$; and these equations give

$$
\cot z \frac{d \Omega}{d \phi}=\cot \phi \frac{d \Omega}{d z}-\operatorname{cosec} \phi \frac{d \Omega}{d \theta},
$$

an equation which is satisfied by $\Omega=\Omega(r, z, \theta, \phi)$. We have thus

$$
\begin{array}{rlrl}
d r & =0, & & \\
d v & =0, & & \\
d y & =0, & & \\
d \frac{n a e \sin f}{\sqrt{1-e^{2}}} & = & & \frac{d \Omega}{d r} d t, \\
d n a^{2} \sqrt{1-e^{2}} & = & \frac{d \Omega}{d r} d t, \\
d \phi & =\frac{\cot z}{n a^{2} \sqrt{1-e^{2}}} \frac{d \Omega}{d \phi} d t,
\end{array}
$$


which may be replaced by

$$
\begin{aligned}
d r & =0, \\
d \theta & =\frac{\operatorname{cosec} \phi}{n a^{2} \sqrt{1-e^{2}}} \frac{d \Omega}{d \phi} d t, \\
d z & =\frac{-\cot \phi}{n a^{2} \sqrt{1-e^{2}}} \frac{d \Omega}{d \phi} d t, \\
d \frac{n a e \sin f}{\sqrt{1-e^{2}}} & = \\
d n a^{2} \sqrt{1-e^{2}} & = \\
d \phi & =\frac{d \Omega}{d r} d t, \\
n a^{2} \sqrt{1-e^{2}} & \frac{d \Omega}{d z} d t,
\end{aligned}
$$

where as before $\Omega=\Omega(r, z, \theta, \phi)$.

I remark that in the case of any central force whatever, we have an element $h$ corresponding to $n a^{2} \sqrt{1-e^{2}}$ in the elliptic theory, and the system for the variations is

$$
\begin{aligned}
d r & =0 \\
d \theta & =\frac{\operatorname{cosec} \phi}{h} \frac{d \Omega}{d \phi} d t \\
d z & =\frac{-\cot \phi}{h} \frac{d \Omega}{d \phi} d t \\
d \frac{d r}{d t} & =\frac{d \Omega}{d r} d t \\
d h & =\frac{d \Omega}{d z} d t \\
d \phi & =\frac{\cot z}{h} \frac{d \Omega}{d \phi} d t
\end{aligned}
$$

where $\Omega=\Omega(r, z, \theta, \phi)$.

Imagine a point in the orbit, which I call the departure-point, the angular distances from this point are termed departures. And I write

so that we have

$p$, the departure of planet,

$\varpi$, the departure of pericentre,

$\sigma$, the departure of node,

$$
\begin{aligned}
& p=\sigma+f, \\
& z=p-\sigma, \\
& \tau=\varpi-\sigma .
\end{aligned}
$$


I write also

$\curlyvee$, the longitude in orbit of departure-point, or, as it may be termed, the adjustment;

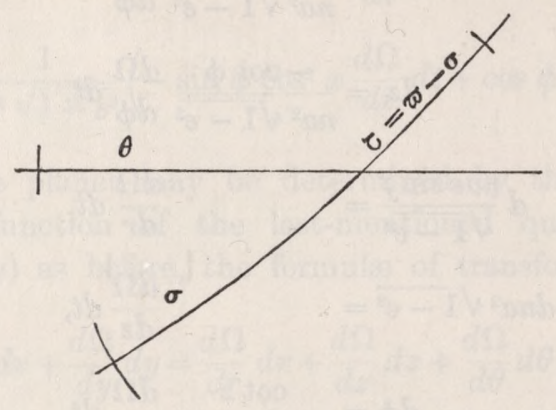

that is

$$
\gamma=\theta-\sigma .
$$

In the undisturbed motion the departure-point is simply a fixed point in the orbit, but when the orbit is variable, the departure-point is taken to be the point of intersection of the orbit with any orthogonal trajectory of the successive positions of the orbit, a definition which is expressed analytically by the equation,

$$
d \sigma=\cos \phi d \theta
$$

The equation, $z=p-\sigma$, gives

$$
d z=d p-d \sigma=d p-\cos \phi d \theta
$$

or, what is the same thing,

$$
d p=d z+\cos \phi d \theta
$$

But we have $d z+\cos \phi d \theta=0$, and consequently $d p=0$, an equation which expresses that the increment of departure, in so far as such increment arises from the variation of the elements, is equal to zero. Or, what is the same thing, the total increment of departure is equal to the infinitesimal angle between two consecutive radius vectors of the planet.

I propose to consider the departure-point as a point which is constantly defined as above, viz., when the orbit is variable, the departure-point is the point of intersection of the orbit with any orthogonal trajectory of the successive positions of the orbit; and as a particular case of the definition, when the orbit is fixed, the departure-point is simply a fixed point on the orbit. The orbit here considered is that of the planet and the position of the planet is determined by the departure and radius vector (the latitude being zero), and this is assumed to be the case whenever the departure is spoken of, and it is such departure which is denoted by the letter p. But we might consider a departure-point (defined as above), upon any other orbit whatever, and use such departure-point as an origin of longitude (for instance, in the lunar theory we might consider a longitude measured along the variable plane of the 
sun's orbit from a departure-point, defined as above, in that orbit), and the position of the planet would then be determined by means of the longitude, latitude, and radius vector. The term sidereal longitude is, I think, used in Physical Astronomy rather loosely to denote the longitude in the mean ecliptic from the mean equinox, less the precession; so defined it is not practically different from, and may I think in all cases be replaced by the longitude as measured from a departure-point in the mean ecliptic.

Returning from this digression, the assumed equation, $d \sigma=\cos \phi d \theta$, gives the expression for the variation $d \sigma$ of the departure of the node, and we now have in the place of the former six equations the seven equations

$$
\begin{array}{rlrl}
d r & =0, & \\
d p & =0, & \\
d \frac{\gamma e \sin f}{\sqrt{1-e^{2}}} & = & \frac{d \Omega}{d r} d t, \\
d n a^{2} \sqrt{1-e^{2}} & = \\
d \phi & =\frac{\cot z}{n a^{2} \sqrt{1-e^{2}}} \frac{d \Omega}{d \phi} d t, \\
d \sigma & =\frac{\cot \phi}{n a^{2} \sqrt{1-e^{2}}} \frac{d \Omega}{d \phi} d t, \\
d \theta & =\frac{\operatorname{cosec} \phi}{n a^{2} \sqrt{1-e^{2}}} \frac{d \Omega}{d \phi} d t,
\end{array}
$$

where as before $\Omega=\Omega(r, z, \theta, \phi)$.

But the value of $z$ is $z=p-\sigma$, and $\Omega$ can be expressed, and that in a single way only, viz. by means of the substitution of $p-\sigma$ in the place of $z$, in the form $\Omega=\Omega(r, p, \sigma, \theta, \phi)$, and if on the right-hand side $\Omega=\Omega(r, z, \theta, \phi)$ as before, then we have

$$
\begin{array}{r}
\frac{d \Omega}{d r}=\frac{d \Omega}{d r}, \\
\frac{d \Omega}{d p}=\frac{d \Omega}{d z}, \\
-\frac{d \Omega}{d \sigma}=\frac{d \Omega}{d z}, \\
\frac{d \Omega}{d \phi}=\frac{d \Omega}{d \phi}, \\
\frac{d \Omega}{d \theta}=\frac{d \Omega}{d \theta},
\end{array}
$$


where on the left-hand side $\Omega=\Omega(r, p, \sigma, \theta, \phi)$. The function $\Omega$ so expressed satisfies, of course, the partial differential equation

$$
\frac{d \Omega}{d p}+\frac{d \Omega}{d \sigma}=0
$$

(which conversely implies that $p, \sigma$ only enters through the function $p-\sigma$ ), and it also satisfies the partial differential equation obtained from the before-mentioned equation

$$
\cot z \frac{d \Omega}{d \phi}=\cot \phi \frac{d \Omega}{d z}-\operatorname{cosec} \phi \frac{d \Omega}{d \theta}, \quad(\Omega=\Omega(r, z, \theta, \phi)),
$$

by the introduction of the transformed expressions of the differential coefficients, and which may be written

$$
\cot z \frac{d \Omega}{d \phi}=-\cot \phi \frac{d \Omega}{d \sigma}-\operatorname{cosec} \phi \frac{d \Omega}{d \theta}
$$

where $\Omega=\Omega(r, \mathrm{p}, \sigma, \theta, \phi)$.

Using the last-mentioned equation to transform the value of $d \phi$, the expressions for the variations become

$$
\begin{aligned}
d r & =0 \\
d p & =0 \\
d \frac{n a e \sin f}{\sqrt{1-e^{2}}} & =\frac{d \Omega}{d r} d t \\
d n a^{2} \sqrt{1-e^{2}} & =\frac{d \Omega}{d p} d t \\
d \phi & =\frac{-\cot \phi}{n a^{2} \sqrt{1-e^{2}}} \frac{d \Omega}{d \sigma} d t-\frac{\operatorname{cosec} \phi}{n a^{2} \sqrt{1-e^{2}}} \frac{d \Omega}{d \theta} d t \\
d \sigma & =\frac{\cot \phi}{n a^{2} \sqrt{1-e^{2}}} \frac{d \Omega}{d \phi} d t \\
d \theta & =\frac{\operatorname{cosec} \phi}{n a^{2} \sqrt{1-e^{2}}} \frac{d \Omega}{d \phi} d t
\end{aligned}
$$

where $\Omega=\Omega(r, p, \sigma, \theta, \phi)$ as before.

I suppose now that the orbit of the planet, instead of being referred to a fixed plane, is referred to a moveable plane or orbit of reference. It is assumed that the longitudes in the orbit of reference are measured from a departure-point defined as above,-that is, from the point in which the orbit of reference is intersected by any orthogonal trajectory of the successive positions of the orbit of reference. And the 
position in regard to the fixed plane, of the orbit of reference, and of the departurepoint in this orbit, are determined by $\theta^{\prime}, \sigma^{\prime}, \phi^{\prime}$,- that is, we have for the orbit of reference,

$$
\begin{aligned}
& \theta^{\prime} \text {, the longitude of node, } \\
& \sigma^{\prime} \text {, the departure of node, } \\
& \phi^{\prime}, \text { the inclination. }
\end{aligned}
$$

The position of the planet's orbit in relation to the moveable orbit of reference is determined in like manner by $\Theta, \Sigma, \Phi$,-that is, we have for the planet's orbit in relation to the orbit of reference,
$\Theta$, the longitude of node,
$\Sigma$, the departure of node,
$\Phi$, the inclination.

Hence if, as before, $\theta, \sigma, \phi$, belong to the orbit of the planet considered in relation to the fixed plane, $\Sigma-\sigma, \Theta-\sigma^{\prime}, \theta-\theta^{\prime}$, will be the sides of a spherical triangle, the opposite angles of which are $\phi^{\prime}, 180^{\circ}-\phi$ and $\Phi$.

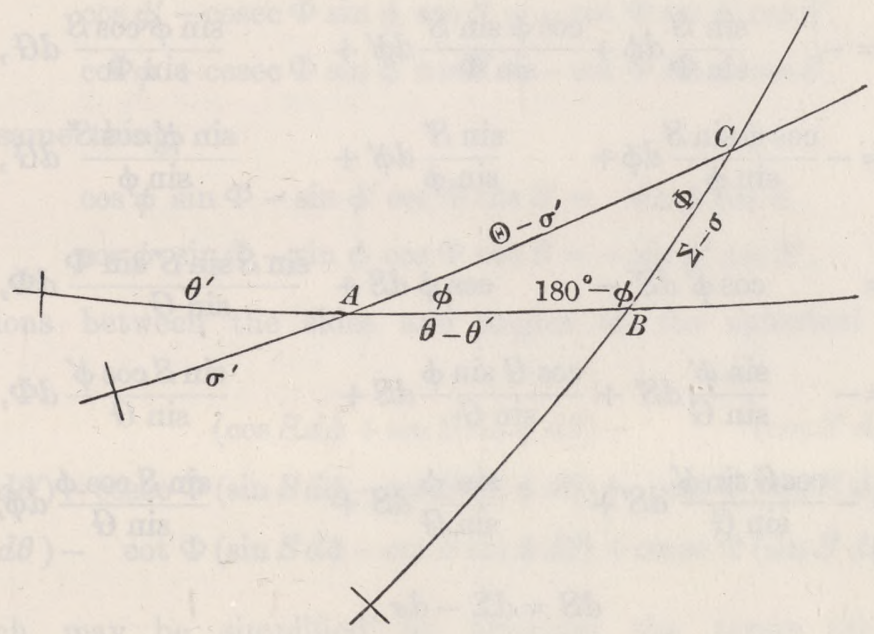

Putting for shortness $S=\Sigma-\sigma, S^{\prime \prime}=\Theta-\sigma^{\prime}, G=\theta-\theta^{\prime}$, so that these symbols denote $S$, the distance of node, along planet's orbit, from fixed plane, $S^{\prime}$, the distance of node, along orbit of reference, from fixed plane, $G$, the distance in fixed plane of the nodes on fixed plane, the sides of the spherical triangle are $S, S^{\prime}, G$, and the opposite angles are $\phi^{\prime}, 180^{\circ}-\phi, \Phi$. C. III. 
Calling the sides $A, B, C$, and the opposite angles $a, b, c$, the general formulæ for a spherical triangle give without difficulty,

$$
\begin{aligned}
& d C=-\cos b d A-\quad \cos a d B+\frac{\sin A \sin B \sin c}{\sin C} d c \\
& d a=\frac{\sin b}{\sin C} d A+\frac{\cos C \sin a}{\sin C} d B+\frac{\sin A \cos b}{\sin C} d c \\
& d b=\frac{\cos C \sin b}{\sin C} d A+\frac{\sin a}{\sin C} d B+\frac{\sin B \cos a}{\sin C} d c
\end{aligned}
$$

and conversely

$$
\begin{aligned}
& d c=\quad \cos B d a+\quad \cos A d b+\frac{\sin a \sin b \sin C}{\sin C} d C \\
& d A=\quad \frac{\sin B}{\sin c} d a-\frac{\cos c \sin A}{\sin c} d b-\frac{\sin a \cos B}{\sin c} d C \\
& d B=-\frac{\cos c \sin B}{\sin c} d a+\quad \frac{\sin A}{\sin c} d b-\frac{\sin b \cos A}{\sin c} d C
\end{aligned}
$$

which, in the present case, become

$$
\begin{aligned}
& d \Phi=\quad \cos S d \phi-\quad \cos S^{\prime \prime} d \phi^{\prime}+\frac{\sin \phi \sin \phi^{\prime} \sin G}{\sin \Phi} d G \\
& d S^{\prime \prime}=-\frac{\sin S}{\sin \Phi} d \phi+\frac{\cos \phi \sin S^{\prime \prime}}{\sin \Phi} d \phi^{\prime}+\frac{\sin \phi \cos S}{\sin \Phi} d G \\
& d S=-\frac{\cos \phi \sin S}{\sin \phi} d \phi+\frac{\sin S^{\prime \prime}}{\sin \phi} d \phi^{\prime}+\frac{\sin S^{\prime \prime}}{\sin \phi} d G
\end{aligned}
$$

and

$$
\begin{aligned}
& d G=\quad \cos \phi^{\prime} d S^{\prime \prime}-\quad \cos \phi d S+\frac{\sin S \sin S^{\prime} \sin \Phi}{\sin G} d \Phi, \\
& d \phi=-\frac{\sin \phi^{\prime}}{\sin G} d S^{\prime}+\frac{\cos G \sin \phi}{\sin G} d S+\quad \frac{\sin S \cos \phi^{\prime}}{\sin G} d \Phi, \\
& d \phi^{\prime}=-\frac{\cos G \sin \phi^{\prime}}{\sin G} d S^{\prime}+\frac{\sin \phi}{\sin G} d S+\frac{\sin S \cos \phi}{\sin G} d \phi,
\end{aligned}
$$

and we have also

$$
\begin{aligned}
& d S=d \Sigma-d \sigma, \\
& d S^{\prime}=d \Theta-d \sigma^{\prime}, \\
& d G=d \theta-d \theta^{\prime} .
\end{aligned}
$$
written

Hence, observing that $\sin S \sin \phi=\sin S^{\prime} \sin \phi^{\prime}$, the preceding equations may be

$$
\begin{aligned}
& d \Phi=\cos S d \phi-\quad \cos S^{\prime} d \phi^{\prime}+\sin S \sin \phi d \theta-\sin S^{\prime} \sin \phi^{\prime} d \theta^{\prime} \\
& d \Theta=d \sigma^{\prime}-\operatorname{cosec} \Phi \sin S d \phi+\cot \phi \sin S^{\prime} d \phi^{\prime}+\operatorname{cosec} \Phi \sin \phi^{\prime} \cos S\left(d \theta-d \theta^{\prime}\right), \\
& d \Sigma=d \sigma-\cot \Phi \sin S d \phi+\operatorname{cosec} \phi \sin S^{\prime} d \phi^{\prime}+\operatorname{cosec} \Phi \sin \phi^{\prime} \cos S^{\prime}\left(d \theta-d \theta^{\prime}\right),
\end{aligned}
$$


and

$$
d \theta=d \theta^{\prime}+\quad \cos \phi^{\prime}\left(d \Theta-d \sigma^{\prime}\right)-\quad \cos \phi(d \Sigma-d \sigma)+\quad \sin S \sin \phi d \Phi,
$$

$d \phi=-\operatorname{cosec} G \sin \phi^{\prime}\left(d \Theta-d \sigma^{\prime}\right)+\cot G \sin \phi(d \Sigma-d \sigma)+\operatorname{cosec} G \sin S^{\prime \prime} \cos \phi^{\prime} d \Phi$,

$d \phi^{\prime}=-\cot G \sin \phi^{\prime}\left(d \Theta-d \sigma^{\prime}\right)+\operatorname{cosec} G \sin \phi(d \Sigma-d \sigma)+\operatorname{cosec} G \sin S \cos \phi d \Phi$,

and it is proper to remark, that in obtaining these equations no use has been made of the equations $d \sigma=\cos \phi d \theta, d \sigma^{\prime}=\cos \phi^{\prime} d \theta^{\prime}$.

The term in $d \Theta$ which contains $d \sigma^{\prime}$, \&c. may be written

$$
\left(d \sigma^{\prime}-\cos \phi^{\prime} d \theta^{\prime}\right)+\cos \phi^{\prime} d \theta^{\prime}+\cot \Phi \sin S^{\prime} d \phi^{\prime}-\operatorname{cosec} \Phi \sin \phi \cos S d \theta^{\prime},
$$

which is equal to

$$
\left(d \sigma^{\prime}-\cos \phi^{\prime} d \theta^{\prime}\right)+\cot \Phi\left(\sin S^{\prime} d \phi^{\prime}-\cos S^{\prime \prime} \sin \phi^{\prime} d \theta^{\prime}\right) ;
$$

and the term in $d \Sigma$ which contains $d \sigma$, \&c. may be written

$$
(d \sigma-\cos \phi d \theta)+\cos \phi d \theta-\cot \Phi \sin S d \phi+\operatorname{cosec} \Phi \sin \phi^{\prime} d \theta,
$$

which is equal to

$$
(d \sigma-\cos \phi d \theta)-\cot \Phi(\sin S d \phi-\cos S \sin \phi d \theta) ;
$$

reductions which depend on

$$
\begin{aligned}
& \cos \phi^{\prime}-\operatorname{cosec} \Phi \sin \phi \cos S=-\cot \Phi \sin \phi^{\prime} \cos S^{\prime}, \\
& \cos \phi+\operatorname{cosec} \Phi \sin \phi^{\prime} \cos S^{\prime}=-\cot \Phi \sin \phi \cos S,
\end{aligned}
$$

or, what is the same thing,

$$
\begin{aligned}
& \cos \phi^{\prime} \sin \Phi-\sin \phi^{\prime} \cos \Phi \cos S^{\prime}=\sin \phi \cos S, \\
& \cos \phi \sin \Phi-\sin \phi \cos \Phi \cos S=-\sin \phi^{\prime} \cos S^{\prime \prime},
\end{aligned}
$$

which are relations between the sides and angles of the spherical triangle. And we then have

$d \Phi=$

$d \Theta=\left(d \sigma^{\prime}-\cos \phi^{\prime} d \theta^{\prime}\right)-\operatorname{cosec} \Phi(\sin S d \phi-\cos S \sin \phi d \theta)+\cot \Phi\left(\sin S^{\prime} d \phi^{\prime}-\cos S^{\prime} \sin \phi^{\prime} d \theta^{\prime}\right)$, $d \Sigma=(d \sigma-\cos \phi d \theta)-\cot \Phi(\sin S d \phi-\cos S \sin \phi d \theta)+\operatorname{cosec} \Phi\left(\sin S^{\prime} d \phi^{\prime}-\cos S^{\prime} \sin \phi^{\prime} d \theta^{\prime}\right)$, expressions which may be simplified by omitting the terms $\left(d \sigma-\cos \phi^{\prime} d \theta^{\prime}\right)$ and $(d \sigma-\cos \phi d \theta)$.

Next substituting for $d \sigma, d \phi, d \theta$, their values, we obtain,

$$
\begin{array}{ll}
d r & =0 \\
d p & =0 \\
d \frac{n a e \sin f}{\sqrt{1-e^{2}}} & =\frac{d \Omega}{d r} d t
\end{array}
$$


$d n a^{2} \sqrt{1-e^{2}}=\frac{d \Omega}{d p} d t$,

$d \Phi=\frac{1}{n a^{2} \sqrt{1-e^{2}}}\left(\sin S \frac{d \Omega}{d \phi}-\cos S\left(\cot \phi \frac{d \Omega}{d \sigma}+\operatorname{cosec} \phi \frac{d \Omega}{d \theta}\right)\right) d t-\quad\left(\sin S^{\prime} d \phi^{\prime}+\sin S^{\prime} \sin \phi^{\prime} d \theta^{\prime}\right)$

$d \Sigma=\frac{\cot \Phi}{n a^{2} \sqrt{1-e^{2}}}\left(\cos S \frac{d \Omega}{d \phi}+\sin S\left(\cot \phi \frac{d \Omega}{d \sigma}+\operatorname{cosec} \phi \frac{d \Omega}{d \theta}\right)\right) d t+\operatorname{cosec} \Phi\left(\sin S^{\prime} d \phi^{\prime}-\cos S^{\prime} \sin \phi^{\prime} d \theta^{\prime}\right)$,

$d \Theta=\frac{\operatorname{cosec} \Phi}{n a^{2} \sqrt{1-e^{2}}}\left(\cos S \frac{d \Omega}{d \phi}+\sin S\left(\cot \phi \frac{d \Omega}{d \sigma}+\operatorname{cosec} \phi \frac{d \Omega}{d \theta}\right)\right) d t+\cot \Phi\left(\sin S^{\prime} d \phi^{\prime}-\cos S^{\prime} \sin \phi^{\prime} d \theta^{\prime}\right)$,

where $\Omega=\Omega(r, p, \sigma, \theta, \phi)$, as before.

But $\Omega$ may be expressed in the form $\Omega=\Omega\left(r, p, \Sigma, \Theta, \Phi, \sigma^{\prime}, \theta^{\prime}, \phi^{\prime}\right)$, or disregarding $\sigma^{\prime}, \theta^{\prime}, \phi^{\prime}$, in the form $\Omega=\Omega(r, p, \Sigma, \Theta, \Phi)$, and to effect the transformation of the differential coefficients we must write,

$$
\begin{aligned}
& d \Phi=\cos S d \phi+\sin S \sin \phi d \theta \\
& d \Theta=-\operatorname{cosec} \Phi(\sin S d \phi-\cos S \sin \phi d \theta) \\
& d \Sigma=(d \sigma-\cos \phi d \theta)-\cot \Phi(\sin S d \phi-\cos S \sin \phi d \theta)
\end{aligned}
$$

or, what is the same thing,

$$
\begin{aligned}
& d \phi=\cos S d \phi-\sin S \sin \Phi d \Theta \\
& d \theta=\quad \operatorname{cosec} \phi(\sin S d \Phi+\cos S \sin \Phi d \Theta) \\
& d \sigma=d \Sigma-\cos \Phi d \Theta+\cot \phi(\sin S d \Phi+\cos S \sin \Phi d \Theta)
\end{aligned}
$$

and substituting in

$$
\begin{aligned}
& \frac{d \Omega}{d r} d r+\frac{d \Omega}{d p} d p+\frac{d \Omega}{d \sigma} d \sigma+\frac{d \Omega}{d \theta} d \theta+\frac{d \Omega}{d \phi} d \phi \\
= & \frac{d \Omega}{d r} d r+\frac{d \Omega}{d p} d p+\frac{d \Omega}{d \Sigma} d \Sigma+\frac{d \Omega}{d \Theta} d \Theta+\frac{d \Omega}{d \Phi} d \Phi,
\end{aligned}
$$

if on the right-hand side $\Omega=\Omega(r, p, \sigma, \theta, \phi)$ as before, then we have

$$
\begin{aligned}
& \frac{d \Omega}{d r}=\frac{d \Omega}{d r}, \\
& \frac{d \Omega}{d p}=\frac{d \Omega}{d p}, \\
& \frac{d \Omega}{d \Sigma}=\frac{d \Omega}{d \sigma}, \\
& \frac{d \Omega}{d \Theta}=(-\cos \Phi+\cot \phi \cos S \sin \Phi) \frac{d \Omega}{d \sigma}+\operatorname{cosec} \phi \cos S \sin \Phi \frac{d \Omega}{d \theta}-\sin S \sin \Phi \frac{d \Omega}{d \phi}, \\
& \frac{d \Omega}{d \Sigma}=\quad \cot \phi \sin S \frac{d \Omega}{d \sigma}+\quad \operatorname{cosec} \phi \sin S \frac{d \Omega}{d \theta}+\quad \cos S \frac{d \Omega}{d \phi},
\end{aligned}
$$

where on the left-hand side $\Omega=\Omega(r, p, \Sigma, \Theta, \Phi)$. 
The last three equations give

$$
\begin{aligned}
\cos S \frac{d \Omega}{d \phi}+\sin S\left(\cot \phi \frac{d \Omega}{d \sigma}+\operatorname{cosec} \phi \frac{d \Omega}{d \theta}\right) & =\frac{d \Omega}{d \Phi}, \\
-\sin S \frac{d \Omega}{d \phi}+\cos S\left(\cot \phi \frac{d \Omega}{d \sigma}+\operatorname{cosec} \phi \frac{d \Omega}{d \theta}\right) & =\cot \Phi \frac{d \Omega}{d \Sigma}+\operatorname{cosec} \Phi \frac{d \Omega}{d \Theta},
\end{aligned}
$$

and the formulæ for the variations become

$$
\begin{aligned}
& d r \quad=0 \text {, } \\
& d p \quad=0 \text {, } \\
& d \frac{n a e \sin f}{\sqrt{1-e^{2}}}=\frac{d \Omega}{d r} d t \\
& d n a^{2} \sqrt{1-e^{2}}=\frac{d \Omega}{d p} d t \\
& d \Phi=\frac{-\cot \Phi}{n a^{2} \sqrt{1-e^{2}}} \frac{d \Omega}{d \Sigma} d t-\frac{\operatorname{cosec} \Phi}{n a^{2} \sqrt{1-e^{2}}} \frac{d \Omega}{d \Theta} d t-\left(\cos S^{\prime} d \phi^{\prime}+\sin S^{\prime \prime} \sin \phi^{\prime} d \theta^{\prime}\right), \\
& d \Sigma \quad=\frac{\cot \Phi}{n a^{2} \sqrt{1-e^{2}}} \frac{d \Omega}{d \Phi} d t \quad+\operatorname{cosec} \Phi\left(\sin S^{\prime} d \phi^{\prime}-\cos S^{\prime} \sin \phi^{\prime} d \theta^{\prime}\right), \\
& d \Theta \quad=\frac{\operatorname{cosec} \Phi}{n a^{2} \sqrt{1-e^{2}}} d \Phi \text { d } d t \quad+\cot \Phi\left(\sin S^{\prime} d \phi^{\prime}-\cos S^{\prime} \sin \phi^{\prime} d \theta^{\prime}\right),
\end{aligned}
$$

where $\Omega=\Omega(r, p, \Sigma, \Theta, \Phi)$. It will be recollected that the value of $S^{\prime}$ is $=\Theta-\sigma^{\prime}$. It may be noticed that

$$
d \Sigma-\cos \Phi d \Theta=\sin \Phi\left(\sin S^{\prime} d \phi^{\prime}-\cos S^{\prime} \sin \phi^{\prime} d \theta^{\prime}\right) .
$$

The system just obtained is, except as regards the terms involving $d \phi^{\prime}$ and $d \theta^{\prime}$, precisely similar in its form to that in which the planet is referred to a fixed plane, or where $\Omega=\Omega(r, p, \sigma, \theta, \phi)$, and this is of course as it should be.

We have now

$$
\begin{aligned}
& p=\varpi+f, \\
& r=a \text { elqr }(e, g), \\
& f=\operatorname{elta}(e, g),
\end{aligned}
$$

so that the position of the planet is determined by means of the elements $a, e, g, \varpi, \Sigma, \Theta, \Phi$. To find the variations of these elements, substituting for $r$ its value in terms of $f$, the first, third, and fourth equations are

$$
\begin{aligned}
& d \frac{a\left(1-e^{2}\right)}{1+e \cos f}=0, \\
& d \frac{n a e \sin f}{\sqrt{1-e^{2}}}=\frac{d \Omega}{d r} d t, \\
& d n a^{2} \sqrt{1-e^{2}}=\frac{d \Omega}{d p} d t,
\end{aligned}
$$


which give

$$
\begin{array}{rlrl}
e \sin f d f-\frac{2 e+\left(1+e^{2}\right) \cos f}{1-e^{2}} d e+\frac{1+e \cos f}{a} d a & =0 \\
e \cos f d f+ & \sin f d e & =\frac{\sqrt{1-e^{2}}}{n a} \frac{d \Omega}{d r} d t+\frac{n a^{2} \sqrt{1-e^{2}}}{e \sin f} \frac{d \Omega}{d p} d t \\
- & d e+\frac{1-e^{2}}{2 a e} d a & & \frac{\sqrt{1-e^{2}}}{n a^{2} e} \frac{d \Omega}{d p} d t,
\end{array}
$$

and we thence obtain

$$
\begin{aligned}
& d a=\frac{2 e \sin f}{n \sqrt{1-e^{2}}} \quad \frac{d \Omega}{d r} d t+\frac{2(1+e \cos f)^{2}}{n a\left(1-e^{2}\right)^{\frac{8}{2}}} \frac{d \Omega}{d p} d t \\
& d e=\frac{\sqrt{1-e^{2}} \sin f}{n a} \frac{d \Omega}{d r} d t+\frac{e+2 \cos f+e \cos ^{2} f}{n a^{2} \sqrt{1-e^{2}}} \frac{d \Omega}{d p} d t \\
& d f=\frac{n \sqrt{1-e^{2} \cos f}}{n a e} \frac{d \Omega}{d r} d t-\frac{(2+e \cos f) \sin f}{n a^{2} \sqrt{1-e^{2}}} \frac{d \Omega}{d p} d t
\end{aligned}
$$

the last of which equations, combined with

gives

$$
d f=\frac{(1+e \cos f)^{2}}{\left(1-e^{2}\right)^{\frac{3}{2}}} d g+\frac{(2+e \cos f) \sin f}{1-e^{2}} d e,
$$

$$
d g=\frac{\left(1-e^{2}\right)\left(-2 e+\cos f+e \cos ^{2} f\right)}{n a e(1+e \cos f)} \frac{d \Omega}{d r} d t-\frac{(2+e \cos f) \sin f}{n a^{2} e} \frac{d \Omega}{d p} d t .
$$

The fourth equation of the formulæ for the variations, viz., $d p=0$, gives $0=d \omega+d f$, and therefore $d \omega=-d f$, that is,

$$
d \omega=-\frac{\sqrt{1-e^{2}} \cos f}{n a e} \frac{d \Omega}{d r} d t+\frac{(2+e \cos f) \sin f}{n a^{2} \sqrt{1-e^{2}}} \frac{d \Omega}{d p} d i
$$

and the complete system becomes therefore

$$
\begin{array}{ll}
d a= & \frac{2 e \sin f}{n \sqrt{1-e^{2}}} \frac{d \Omega}{d r} d t+ \\
d e= & \frac{\sqrt{1-e^{2}} \sin f}{n a} \frac{d \Omega}{n a\left(1-e^{2}\right)^{\frac{3}{2}}} \frac{d \Omega}{d p} d t, \\
d g=\frac{\left(1-e^{2}\right)\left(-2 e+\cos f+e \cos ^{2} f\right)}{n a e(1+e \cos f)} \frac{d \Omega}{d r} d t-\frac{e+2 \cos f+e \cos ^{2} f}{n a^{2} \sqrt{1-e^{2}}} \frac{d \Omega}{d p} d t, & \frac{(2+e \cos f) \sin f}{n a^{2} e} \frac{d \Omega}{d p} d t, \\
d \sigma= & -\frac{\sqrt{1-e^{2}} \sin f}{n a e} \frac{d \Omega}{d r} d t+\frac{(2+e \cos f) \sin f}{n a^{2} e} \frac{d \Omega}{d p} d t,
\end{array}
$$




$$
\begin{array}{ll}
d \Phi=\frac{-\cot \Phi}{n a^{2} \sqrt{1-e^{2}}} \frac{d \Omega}{d \Sigma} d t-\frac{\operatorname{cosec} \Phi}{n a^{2} \sqrt{1-e^{2}}} \frac{d \Omega}{d \Theta} d t & \left(\cos S^{\prime \prime} d \phi^{\prime}+\sin S^{\prime} \sin \phi^{\prime} d \theta^{\prime}\right), \\
d \Sigma=\frac{\cot \Phi}{n a^{2} \sqrt{1-e^{2}}} \frac{d \Omega}{d \Phi} d t & +\operatorname{cosec} \Phi\left(\sin S^{\prime} d \phi^{\prime}-\cos S^{\prime \prime} \sin \phi^{\prime} d \theta^{\prime}\right), \\
d \Theta=\frac{\operatorname{cosec} \Phi}{n a^{2} \sqrt{1-e^{2}}} \frac{d \Omega}{d \Phi} d t & +\cot \Phi\left(\sin S^{\prime \prime} d \phi^{\prime}-\cos S^{\prime \prime} \sin \phi^{\prime} d \theta^{\prime}\right),
\end{array}
$$

where, as before, $\Omega=\Omega(r, p, \Sigma, \Theta, \Phi)$. This is the first form of the expressions for the variations of the elements.

But we may in the disturbing function $\Omega$ replace $r, p$, by their values in terms of $a, e, g, \varpi$, and if on the right-hand side $\Omega$ has the last preceding value, we have

$$
\begin{gathered}
\frac{d \Omega}{d a} d a+\frac{d \Omega}{d e} d e+\frac{d \Omega}{d g} d g+\frac{d \Omega}{d \Phi} d \varpi+\frac{d \Omega}{d \Sigma} d \Sigma+\frac{d \Omega}{d \Theta} d \Theta+\frac{d \Omega}{d \Phi} d \Phi \\
=\frac{d \Omega}{d r} d r+\frac{d \Omega}{d p} d p+\frac{d \Omega}{d \Sigma} d \Sigma+\frac{d \Omega}{d \Theta} d \Theta+\frac{d \Omega}{d \Phi} d \Phi
\end{gathered}
$$

where on the left-hand side $\Omega=\Omega(a, e, g, \varpi, \Sigma, \Theta, \Phi)$; and the expressions .for the differentials $d r$ and $d p$, are

$$
\begin{aligned}
& d r=\frac{1-e^{2}}{1+e \cos f} d a-a \cos f d t+\frac{a e \sin f}{\sqrt{1-e^{2}}} d g \\
& d p=\frac{(2+e \cos f) \sin f}{1-e^{2}} d e+\frac{(1+e \cos f)^{2}}{(1-e)^{\frac{3}{2}}} d g+d \omega
\end{aligned}
$$

and we have therefore

$$
\begin{array}{lc}
\frac{d \Omega}{d a}= & \frac{1-e^{2}}{1+e \cos f} \frac{d \Omega}{d r}, \\
\frac{d \Omega}{d e}= & -a \cos f \frac{d \Omega}{d r}+\frac{(2+e \cos f) \sin f}{1-e^{2}} \frac{d \Omega}{d p}, \\
\frac{d \Omega}{d g}= & \frac{a e \sin f}{\sqrt{1-e^{2}}} \frac{d \Omega}{d r}+\frac{(1+e \cos f)^{2}}{\left(1-e^{2}\right)^{\frac{3}{2}}} \frac{d \Omega}{d p}, \\
\frac{d \Omega}{d \varpi}= & \frac{d \Omega}{d p}, \\
\frac{d \Omega}{d \Phi}= & \frac{d \Omega}{d \Phi}, \\
\frac{d \Omega}{d \Sigma}= & \frac{d \Omega}{d \Sigma}, \\
\frac{d \Omega}{d \Theta}= & \frac{d \Omega}{d \Theta},
\end{array}
$$

where on the left-hand side $\Omega=\Omega(a, e, g, \varpi, \Sigma, \Theta, \Phi)$, 
and we thence obtain for the variations the new system of formulæ,

$$
\begin{aligned}
& d a=+\frac{2}{n a} \frac{d \Omega}{d g} d t \\
& d e=\quad+\frac{1-e^{2}}{n a^{2} e} \frac{d \Omega}{d g} d t-\frac{\sqrt{1-e^{2}}}{n a^{2} e} \frac{d \Omega}{d \sigma} d t, \\
& d g=-\frac{2}{n a} \frac{d \Omega}{d a} d t-\frac{1-e^{2}}{n a^{2} e} \frac{d \Omega}{d e} d t, \\
& d \sigma=\quad+\frac{\sqrt{1-e^{2}}}{n a^{2} e} \frac{d \Omega}{d e} d t, \\
& d \Phi= \\
& \frac{-\cot \Phi}{n a^{2} \sqrt{1-e^{2}}} \frac{d \Omega}{d \Sigma} d t-\frac{\operatorname{cosec} \Phi}{n a^{2} \sqrt{1-e^{2}}} \frac{d \Omega}{d \Theta} d t \\
& \text { - } \quad\left(\cos S^{\prime} d \phi^{\prime}+\sin S^{\prime} \sin \phi^{\prime} d \theta^{\prime}\right), \\
& d \Sigma= \\
& +\frac{\cot \Phi}{n a^{2} \sqrt{1-e^{2}}} \frac{d \Omega}{d \Phi} d t \\
& +\operatorname{cosec} \Phi\left(\sin S^{\prime} d \phi^{\prime}-\cos S^{\prime} \sin \phi^{\prime} d \theta^{\prime}\right), \\
& d \Theta= \\
& +\frac{\operatorname{cosec} \Phi}{n a^{2} \sqrt{1-e^{2}}} \frac{d \Omega}{d \Phi} d t \\
& +\cot \Phi\left(\sin S^{\prime} d \phi^{\prime}-\cos S^{\prime \prime} \sin \phi^{\prime} d \theta^{\prime}\right),
\end{aligned}
$$

where, as before, $\Omega=\Omega(a, e, g, \varpi, \Sigma, \Theta, \Phi)$. This is the second form of the expressions for the variations of the elements. It is hardly necessary to remark, that if in either system of formulæ we omit the terms involving $d \theta^{\prime}$ and $d \phi^{\prime}$, and in the place of $\Sigma, \Theta$, $\Phi$, write $\sigma, \theta, \phi$, we have the formulæ for the variation of the elements when the orbit of the planet is referred to a fixed plane, and the disturbing function is given under the form $\Omega=\Omega(r, p, \sigma, \theta, \phi)$, or $\Omega=\Omega(a, e, g, \sigma, \sigma, \theta, \phi)$.

The demonstration of the two preceding systems forms, as before remarked, the object of the present Memoir. But it is proper to give also the systems for the variations of the elements in the form in which they would have been obtained, if the notion of the departure had not been introduced into the investigation. To do this I revert to a preceding system of equations, which may be written

$$
\begin{array}{ll}
d r & =0 \\
d \theta & =\frac{\operatorname{cosec} \phi}{n a^{2} \sqrt{1-e^{2}}} \frac{d \Omega}{d \phi} d t \\
d z & =\frac{-\cot \phi}{n a^{2} \sqrt{1-e^{2}}} \frac{d \Omega}{d \phi} d t \\
d \frac{n a e \sin f}{\sqrt{1-e^{2}}} & =\quad \frac{d \Omega}{d r} d t
\end{array}
$$




$$
\begin{array}{ll}
d n a^{2} \sqrt{1-e^{2}} & =\frac{d \Omega}{d z} d t, \\
d \phi & =\frac{-\operatorname{cosec} \phi}{n a^{2} \sqrt{1-e^{2}}} \frac{d \Omega}{d \theta} d t+\frac{\cot \phi}{n a^{2} \sqrt{1-e^{2}}} \frac{d \Omega}{d z} d t\left(=\frac{\cot z}{n a^{2} \sqrt{1-e^{2}}} \frac{d \Omega}{d \phi} d t\right),
\end{array}
$$

where $\Omega=\Omega(r, z, \theta, \phi)$.

Substituting in these equations for $r, z$, the values $\frac{a\left(1-e^{2}\right)}{1+e \cos f}$ and $\boldsymbol{\tau}+f$, we obtain

$$
\begin{aligned}
& d a=\frac{2 e \sin f}{n \sqrt{1-e^{2}}} \frac{d \Omega}{d r} d t+\frac{2(1+e \cos f)^{2}}{n a\left(1-e^{2}\right)^{\frac{3}{2}}} \frac{d \Omega}{d z} d t \\
& d e=\frac{\sqrt{1-e^{2}} \sin f}{n a} \frac{d \Omega}{d r} d t+\frac{e+2 \cos f+e \cos ^{2} f}{n a^{2} \sqrt{1-e^{2}}} \frac{d \Omega}{d z} d t \\
& d g=\frac{\left(1-e^{2}\right)\left(-2 e+\cos f+e \cos ^{2} f\right)}{n a e(1+e \cos f)} \frac{d \Omega}{d r} d t-\frac{(2+e \cos f) \sin f}{n a^{2} e} \frac{d \Omega}{d z} d t \\
& d \tau=-\frac{\sqrt{1-e^{2}} \cos f}{n a e} \frac{d \Omega}{d r} d t+\frac{(2+e \cos f) \sin f}{n a^{2} \sqrt{1-e^{2}}} \frac{d \Omega}{d z} d t-\frac{\cot \phi}{n a^{2} \sqrt{1-e^{2}}} \frac{d \Omega}{d \phi} d t \\
& d \phi= \\
& d \theta=
\end{aligned}
$$

where $\Omega=\Omega(r, z, \theta, \phi)$, as before; and which is the first system for the variations. of the six elements, $a, e, g, \tau, \theta, \phi$.

But if in the disturbing function we replace $r, z$ by their values, then if on the left-hand side $\Omega=\Omega(r, z, \theta, \phi)$, as before, we find

$$
\begin{aligned}
\frac{1-e^{2}}{1+e \cos f} \frac{d \Omega}{d r} & =\frac{d \Omega}{d r}, \\
-a \cos f \frac{d \Omega}{d r}+\frac{(2+e \cos f) \sin f}{1-e^{2}} \frac{d \Omega}{d z} & =\frac{d \Omega}{d e}, \\
\frac{a e \sin f}{\sqrt{1-e^{2}}} \frac{d \Omega}{d r}+\frac{(1+e \cos f)^{2}}{\left(1-e^{2}\right)^{\frac{3}{2}}} \frac{d \Omega}{d z} & =\frac{d \Omega}{d g}, \\
\frac{d \Omega}{d \phi} & =\frac{d \Omega}{d \phi}, \\
\frac{d \Omega}{d z} & =\frac{d \Omega}{d \tau}, \\
\frac{d \Omega}{d \theta} & =\frac{d \Omega}{d \theta},
\end{aligned}
$$

where on the right-hand side $\Omega=\Omega(a, e, g, \boldsymbol{\tau}, \theta, \phi)$.

C. III. 
The formulæ for the variations thus become

$d a=$

$$
\frac{2}{n a} \frac{d \Omega}{d g} d t
$$

de $=$

$$
\frac{1-e^{2}}{n a^{2} e} \frac{d \Omega}{d g} d t-\frac{\sqrt{1-e^{2}}}{n a^{2} e} \frac{d \Omega}{d \tau} d t
$$

$d g=-\frac{2}{n a} \frac{d \Omega}{d a} d t-\frac{1-e^{2}}{n a^{2} e} \frac{d \Omega}{d e} d t$

$d \boldsymbol{\tau}=$

$$
\frac{\sqrt{1-e^{2}}}{n a^{2} e} \frac{d \Omega}{d e} d t
$$$$
-\frac{\cot \phi}{n a \sqrt{1-e^{2}}} \frac{d \Omega}{d \phi} d t
$$

$d \phi=$ $\frac{\cot \phi}{n a^{2} \sqrt{1-e^{2}}} \frac{d \Omega}{d \bar{C}} d t$

$-\frac{\operatorname{cosec} \phi}{n a^{2} \sqrt{1-e^{2}}} \frac{d \Omega}{d \theta} d t$

$d \theta=$

$$
+\frac{\operatorname{cosec} \phi}{n a^{2} \sqrt{1-e^{2}}} \frac{d \Omega}{d \phi} d t
$$

where, as before, $\Omega=\Omega(a, e, g, \tau, \theta, \phi)$. This is the second system of formulæ for the variations of the six elements $a, e, g, \tau, \theta, \phi$.

The last-mentioned system may be easily deduced from Jacobi's canonical system of formulæ, viz. putting

$\mathfrak{A}$, the constant of vis viva,

$\mathfrak{B}$, the constant of areas,

(5, the constant of the reduced area,

$\mathfrak{F}$, the constant attached to the time,

(S), the angular distance of pericentre from node,

$\mathfrak{H}$, the longitude of node;

then the canonical system is

$$
\begin{aligned}
& d \mathfrak{A}=\frac{d \Omega}{d \mathfrak{F}} d t, \\
& d \mathfrak{B}=\frac{d \Omega}{d \mathscr{S}} d t, \\
& d \mathfrak{S}=\frac{d \Omega}{d \mathfrak{S}} d t, \\
& d \mathfrak{S}=-\frac{d \Omega}{d \mathfrak{A}} d t, \\
& d \mathscr{S}=-\frac{d \Omega}{d \mathfrak{B}} d t, \\
& d \mathfrak{S}=-\frac{d \Omega}{d \mathfrak{S}} d t,
\end{aligned}
$$


and the expressions for $\mathfrak{A}, \& \mathrm{c}$. in terms of the elements $a, e, g, \boldsymbol{\tau}, \theta, \phi$, are

$$
\begin{aligned}
& \mathfrak{A}=-\frac{1}{2} n^{2} a^{2}, \\
& \mathfrak{B}=n a^{2} \sqrt{1-e^{2}}, \\
& \mathfrak{E}=n a^{2} \sqrt{1-e^{2}} \cos \phi, \\
& \mathfrak{F}=\frac{1}{n} g-t, \\
& \mathfrak{F}=\boldsymbol{C}, \\
& \mathfrak{H}=\theta,
\end{aligned}
$$

and the transformation can be effected without the slightest difficulty.

I shall conclude with the demonstration of a formula which occurs implicitly in Hansen's Lunar Theory, and which may probably be useful for other purposes.

Write

$$
\begin{aligned}
& \rho \text {, the radius vector, } \tau \text { for } t \text {, } \\
& \psi \text {, the true anomaly, } \tau \text { for } t \text {, }
\end{aligned}
$$

that is, let $\rho, \psi$, be what $r, f$, become when the time $t$, in so far as it enters explicitly in $g$, and not through the variable elements, is replaced by an arbitrary quantity, $\tau$.

And suppose, in like manner,

$$
\begin{aligned}
& \gamma \text {, the mean anomaly, } \tau \text { for } t \text {, } \\
& \lambda \text {, the departure, } \tau \text { for } t,
\end{aligned}
$$

and let $l \rho$ denote the logarithm of $\rho$; then we have, attending only to the variation of $t$, in so far as it enters through the variable elements,

$$
\begin{array}{lll}
d f= & \frac{(2+e \cos f) \sin f}{1-e^{2}} d e+ & \frac{(1+e \cos f)^{2}}{\left(1-e^{2}\right)^{\frac{3}{2}}} d g \\
d \psi= & \frac{(2+e \cos \psi) \sin \psi}{1-e^{2}} d e+\quad \frac{(1+e \cos \psi)^{2}}{\left(1-e^{2}\right)^{\frac{3}{2}}} d \gamma \\
d l \rho=\frac{1}{a} d a-\frac{(1+e \cos \psi) \cos \psi}{1-e^{2}} d e+\frac{(1+e \cos \psi) e \sin \psi}{\left(1-e^{2}\right)^{\frac{s}{2}}} d \gamma
\end{array}
$$

We hence deduce,

$$
\begin{aligned}
& d l \rho+\frac{\rho e \sin \psi}{a\left(1-e^{2}\right)}(d f-d \psi) \\
= & \frac{1}{a} d a+\frac{e \sin \psi \sin f(2+e \cos f)-\cos \psi-2 e-e^{2} \cos \psi}{\left(1-e^{2}\right)(1+e \cos \psi)} d e+\frac{e \sin \psi(1+e \cos f)^{2}}{\left(1-e^{2}\right)^{\frac{3}{2}}(1+e \cos \psi)} d g,
\end{aligned}
$$


the coefficient of $d \gamma$ being zero. And substituting for $d a$, $d e, d g$ their values, viz.

$$
\begin{aligned}
& d a= \\
& \frac{2 e \sin f}{n \sqrt{1-e^{2}}} \frac{d \Omega}{d r} d t+ \\
& d e=\quad \frac{2(1+e \cos f)^{2}}{n a \cdot\left(1-e^{2}\right)^{\frac{3}{2}}} \frac{d \Omega}{d p} d t, \\
& d g=\frac{\left(1-e^{2}\right)\left(-2 e+\cos f+e \cos ^{2} f\right)}{n a e(1+e \cos f)} \frac{d \Omega}{d r} d t+\frac{(2+e \cos f) \sin f}{n a^{2} e} \frac{d \Omega}{d p} d t,
\end{aligned}
$$

the equation becomes

$$
\begin{aligned}
d l \rho & +\frac{\rho e \sin \psi}{a\left(1-e^{2}\right)}(d f-d \psi) \\
& =\frac{-\sqrt{1-e^{2}}}{n a(1+e \cos \psi)} \sin (f-\psi) \frac{d \Omega}{d r} d t \\
& +\frac{1}{n a^{2} \sqrt{1-e^{2}}(1+e \cos \psi)}\{2+e \cos \psi-\cos (f-\psi)(2+e \cos f)\} \frac{d \Omega}{d p} d t
\end{aligned}
$$

But we have $p=\sigma+f, \lambda=\sigma+\psi$, and, consequently, $f-\psi=p-\lambda$, and the equation becomes

$$
\begin{aligned}
& d l_{\rho}+\frac{\rho e \sin \psi}{a\left(1-e^{2}\right)}(d p-d \lambda) \\
& =\frac{-\sqrt{1-e^{2}}}{n a(1+e \cos \psi)} \sin (p-\lambda) \frac{d \Omega}{d r} d t \\
& \quad+\frac{1}{n a^{2} \sqrt{1-e^{2}}(1+e \cos \psi)}\{2+e \cos \psi-\cos (p-\lambda)(2+e \cos f)\} \frac{d \Omega}{d p} d t
\end{aligned}
$$

which is the equation referred to; the expression on the right-hand side, omitting the factor $d t$, is, in fact, the portion not involving the arbitrary functions $\Pi, \Gamma$, of Hansen's function $R$ (Fund. p. 43), viz. it is in Hansen's notation,

$$
\begin{aligned}
& -\left\{\frac{\rho}{r} \cos (v,-\lambda)-1+\frac{\rho}{a\left(1-e^{2}\right)}[\cos (v,-\lambda)-1]\right\} \frac{a n}{\sqrt{1-e^{2}}} \frac{d \Omega}{d v} \\
& -\frac{\rho}{r} \sin (v,-\lambda) \frac{a n}{\sqrt{1-e^{2}}} r \frac{d \Omega}{d r},
\end{aligned}
$$

where $a, n, e, r, \rho, v, \lambda, \Omega$ (Hansen), correspond to $a, n, e, r, \rho, p, \lambda, n^{-2} a^{-3} \Omega$, of the present Memoir.

2, Stone Buildings, W. C., 3 March, 1858. 